\title{
Acoustic Characteristics Analysis of Liquid-filled Muffler Based on the Structural Acoustic Coupling
}

\author{
Shuailong Zhou ${ }^{1}$, Zhi Fang ${ }^{2, *}$ \\ ${ }^{1}$ Luoyang Ship Material Research Institute, Luoyang, Henan, China. \\ ${ }^{2}$ School of Naval Architecture and Ocean Engineering, Huazhong University of Science and Technology, Wuhan, Hubei, China.
}

How to cite this paper: Shuailong Zhou, Zhi Fang. (2021) Acoustic Characteristics Analysis of Liquid-filled Muffler Based on the Structural Acoustic Coupling. Journal of Applied Mathematics and Computation, 5(4), 338-348.

DOI: $10.26855 /$ jamc.2021.12.012

Received: November 1, 2021

Accepted: November 26, 2021

Published: December 16, 2021

${ }^{*}$ Corresponding author: Zhi Fang, School of Naval Architecture and Ocean Engineering, Huazhong University of Science and Technology, Wuhan, Hubei, China.

Email: fangzhi@hust.edu.cn

\begin{abstract}
Acoustic-structure coupling effect is an important factor that affects water-filled pipe muffler's acoustic performance. In this paper, finite element method (FEM) was used to study acoustic characteristics of water-filled pipe muffler. Circumferential chamber and perforated pipe walls of the muffler were taken as elastic plates. The effect of acoustic-structure coupling is considered when calculating transmission loss (TL) of water-filled expansion chamber muffler, water mufflers with perforated plate and perforated pipe. In this paper, the effects of the wall thickness and material of the elastic wall and the structural parameters of the perforated plate on the acoustic characteristics of the water muffler are further studied. By comparing the elastic cavity wall of the muffler with the elastic perforated pipe wall, the contribution of both to the acoustic characteristics of the perforated pipe water muffler is obtained.
\end{abstract}

\section{Keywords}

Liquid-filled muffler, Acoustic-structure coupling, Transmission loss

\section{Introduction}

Noise control is essential for ships and submarines, where water-filled mufflers are widely used in marine water piping systems to eliminate vibration and noise. The low-frequency sound absorption performance of the expansion muffler needs to be improved, which is limited by the space size in engineering applications [1-4]. Therefore, the perforated pipe muffler is more widely used because of its smaller flow resistance loss and good sound attenuation [4]. It is the focus of this article to discuss the acoustic characteristics of the water-filled perforated pipe muffler.

There are far more researches on gas mufflers than water mufflers. The acoustic performance of gas mufflers has been investigating a lot and some proven approaches and theories have been applied to predict the (TL) with relatively high credibility, such as the finite element method (FEM) [3, 5, 6], the transfer matrix method (TMM) [7], the finite volume method (FVM) [8], the boundary element method (BEM) [9, 10], analytical method [11, 12]. The research method of gas muffler is applied to water muffler. The muffler principle is basically the same for gas muffler and liquid muffler [13]. In particular, there is a big difference in the interaction between liquid (water, etc.), gas (air, etc.) and the structure of the muffler itself. The coupling between the structure and the gas is extremely small and can be ignored because the structural elasticity of the muffler is much smaller than the compressibility of the gas. In general, the prediction of the acoustic characteristics of gas mufflers does not consider the coupling of structure and gas. On the contrary, the acoustic impedance of the water medium is much larger than that of the gas medium, and its compressibility is closer to the elasticity of the structure. Therefore, the coupling effect of the water medium and the structure should be considered in the process of predicting the acoustic characteristics of the water-filled muffler. Otherwise, the predicted value will have a large devia- 
tion from the experimental value [3]. In this case of thin structures and dense fluids, the feedback of acoustic pressure to structures is not negligible [14].

In the 1970s, the acoustic-structure coupling model was first proposed [15]. After that, Gladwell [16] perfected the acoustic-structure coupling variational equation. It advances the finite element method for solving acoustic-structure coupling problems. Norris and Wickham [17] studied the acoustic scattering of the elastic water Helmholtz resonator, using an asymptotic theory. The research results show that the elastic wall reduced the resonance frequency in comparison with a rigid wall of the same size. Zhou [18] discussed the influence of the elastic branch pipe, main pipe and cavity wall on the acoustic characteristics of the water Helmholtz resonator. The results show that the difference in wall thickness has a significant effect on the acoustic-structure coupling. Based on the research of the elastic Helmholtz resonator, some efforts have been contributed to the water muffler in recent years. For the acoustic problem in dense fluid charmer systems, some theory methods have been developed considering acoustic-structure coupling [19]. Although the research results show the influence of elastic walls on the acoustic characteristics of water mufflers, the model is relatively simple and difficult to be applied when solving practical engineering problems. Taking into account the effect of acoustic-structure coupling, Gong et al. [20] used a numerical model to analyze the internal sound field of the muffler in order to better predict the TL of the water muffler. Numerical results show that the coupling effect is enhanced due to the thinning of the elastic wall. In particular, the transfer loss curve of the expansion chamber muffler shifts to low frequency band, when the acoustic-structure coupling effect of the circumferential wall is considered. In the research of Gong et al. [3] on muffler with water-filled expansion chamber, influence of material properties and elastic walls can be qualitatively analyzed. The sound pressure and the displacement of the elastic wall under acoustic-structure coupling are investigated by COMSOL at different frequencies, which show the acoustic-structure interaction between elastic wall and fluid can be utilized to trigger non-planar waves and increase the number of peaks in the TL curve. However, these efforts excluded the effects of flow and viscosity on the sound propagation and attenuation in the water-filled perforated pipe muffler. Without considering the acoustic-structure coupling, Ji et al. $[13,21]$ and used computational fluid dynamics to analyze the water-filled perforated pipe muffler, and the numerical solution was in good agreement with the theoretical solution or the published experimental date.

Installation of a muffler is proved to be an effective method for hydrodynamic noise control of water-filled pipe systems in practical engineering [2]. To the best knowledge of the authors, on the one hand, few available literatures are found for the acoustic computation and analysis of water-filled perforated pipe muffler, on the other hand, few researches have studied the TL of a water-filled perforated pipe muffler considering the acoustic-structure coupling effect. Then, the acoustic-structure model based on the FEM is employed to investigate acoustic characteristics of water-filled perforated plate and perforated pipe muffler. The objectives of this paper are then to employ the LMS Virtual Lab approach to examine the influence of perforated elastic wall and muffler elastic wall on the sound attenuation behavior of water-filled perforated pipe muffler, which included different position and different thickness parameters.

\section{Numerical simulations}

For coupled acoustic-structure problems, a structural and an acoustic problem need to be solved simultaneously to include the mutual coupling interaction between the acoustic pressure (in water) and the structural deformation (in elastic wall). Generally, the most commonly used way for interior coupled problems, which have a bounded fluid domain, is a coupled finite element-finite element system, in which a structural finite element system is coupled with an acoustic finite element system.

\subsection{Problem definition and boundary condition}

In LMS Virtual Lab, the following four boundary conditions are defined on the finite element model of the muffler:

a) External sound pressure excitation (at the inlet) $\Omega_{\text {in }}$ : The plane wave is set at the entrance of the muffler, whose amplitude is set to be $1 \mathrm{~Pa}$.

$$
\left.P\right|_{\Omega_{\text {in }}}=1 \mathrm{~Pa}
$$

b) Acoustic hard boundary conditions $\Omega_{Z_{\infty}}$ : The sound wave does not consider the sound-solid coupling effect, and the sound speed is 0 , at this boundary.

$$
\left.\mu\right|_{\Omega_{Z_{\infty}}}=0
$$

c) Acoustic impedance boundary conditions $\Omega_{Z t}$ : Indicates the relationship between the sound pressure difference $(\Delta P)$ 
between the upper and lower domains of the perforated pipe (or plate) and the vibration velocity $\left(v_{1}\right)$ of the particle passing through the perforation.

$$
\left.\frac{\Delta P}{\rho_{w} c_{w}}\right|_{\Omega_{Z t}}=Z_{t}
$$

where $Z_{t}, \rho_{w}$ and $c_{w}$ are the acoustic impedance ratio, density of water (set as $1,000 \mathrm{~kg} / \mathrm{m}^{3}$ ) and sound speed in water (set as $1,500 \mathrm{~m} / \mathrm{s}$ ), respectively. Actually, the thickness of the perforation area and the acoustic-structure coupling effect need to be considered, for which a complete finite element model of the perforation needs to be established. The impedance boundary condition is applied to the exit of the muffler to simulate the end of the silencing of the plane wave, which is marked as automatic matching layer (AML) in the software.

d) Acoustic-structure coupling boundary conditions $\Omega_{s}$ : The influence of elastic wall vibration on sound pressure needs to be considered in the numerical calculation, under this boundary condition.

$$
-\vec{n}\left(\frac{1}{-\rho_{w}}\left(\nabla p_{t}-q_{d}\right)\right)=-\left.\vec{n} \cdot \ddot{u} \quad F_{A}\right|_{\Omega_{s}}=p_{t} \cdot \vec{n}
$$

Where $\ddot{u}$ is the acceleration of the structure, $\vec{n}$ is the normal vector of the acoustic-structure coupling surface, $p_{t}$ is the total sound pressure, $F_{A}$ is the surface load on the structure side. Furthermore, the perforated elastic thin wall with sound pressure on both sides, the acoustic-structure coupling boundary equation can be expressed as:

$$
\begin{gathered}
-\vec{n}_{1}\left(\frac{1}{-\rho_{w}}\left(\nabla p_{t 1}-q_{d}\right)\right)=-\vec{n}_{1} \cdot \ddot{u} \\
-\vec{n}_{2}\left(\frac{1}{-\rho_{w}}\left(\nabla p_{t 2}-q_{d}\right)\right)=-\vec{n}_{2} \cdot \ddot{u} \\
\left.F_{A}\right|_{\Omega_{s}}=p_{t 1} \cdot \vec{n}_{1}-p_{t 2} \cdot \vec{n}_{2}
\end{gathered}
$$

In particularly, the subscripts 1 and 2 distinguish the two sides of the structure.

Combining various boundary conditions of structural finite element and acoustic finite element yields an interior coupled acoustic-structure system of the entire model, as shown:

$$
\left[\begin{array}{cc}
K_{s}+j \omega C_{s}-\omega^{2} M_{s} & \bar{Q} \\
\rho_{w} \omega^{2} \bar{Q}^{T} & K_{a}+j \omega C_{a}-\omega^{2} M_{a}
\end{array}\right]\left[\begin{array}{l}
u \\
p
\end{array}\right]=\left[\begin{array}{l}
F_{s} \\
F_{a}
\end{array}\right]
$$

Where $M_{s}, C_{s}$, and $K_{s}$ are structural mass matrix, damping matrix, and stiffness matrix, respectively; $M_{a}, C_{a}$, and $K_{a}$ are fluid mass matrix, damping matrix, and stiffness matrix, respectively, $\bar{Q}$ is the acoustic-structure coupling matrix, $F_{s}$ is the structural load vector, $F_{a}$ is the sound field excitation load vector.

\subsection{Numeral Calculations of TL}

In this paper, the longitudinal sound speed $c_{l}$ and shear sound speed $c_{s}$ in the solid are expressed as:

$$
\begin{aligned}
c_{l} & =\sqrt{\frac{1-v}{(1+v)(1-2 v)}} \sqrt{\frac{E}{\rho}} \\
c_{s} & =\sqrt{\frac{1}{2(1+v)}} \sqrt{\frac{E}{\rho}}
\end{aligned}
$$

where, $E$ is the Young' modulus, $\rho$ is the density and $v$ is the Poisson ratio.

To ensure the accuracy of the analysis, the size of the mesh is needed to be determined based on the highest analysis frequency, and at least six elements are needed within the guaranteed minimum wavelength. In this study, the unit cell is meshed by using unstructured grid, and the size of the elements meets the requirements mentioned above.

When the entire finite element model is established, the sound pressure and vibration velocity at the inlet and outlet of the muffler can be solved by Eq. 9. The cross-sectional area of the outlet and inlet of the muffler are equal, TL can be defined as:

$$
T L=10 \lg \left(\frac{p_{1} \cdot \overline{p_{1}}}{p_{3} \cdot \overline{p_{3}}}\right)
$$




\section{Results and discussion}

\subsection{Water-filled expansion chamber muffler}

The research of the acoustic-structure coupling effect on TL has been published [3, 20], for the water-filled expansion chamber muffler. This section conducts a confirmatory study on this to show the correctness of the research method. The model is shown in Figure 1. The material physical properties and corresponding sound speed are shown in Table 1.

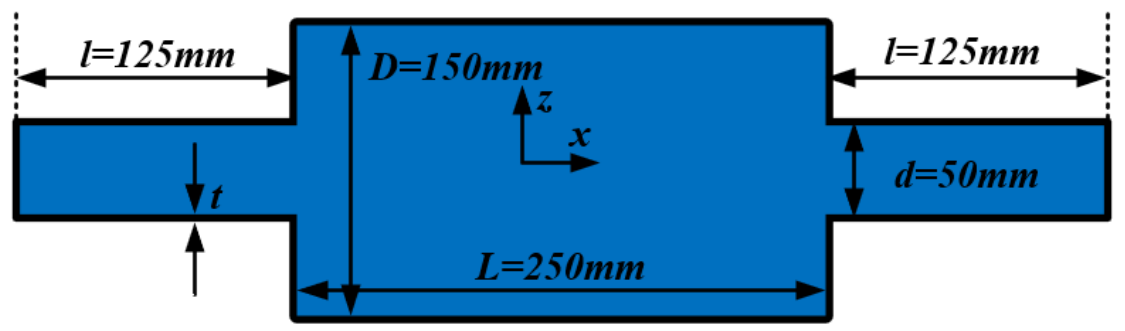

Figure 1. Geometry model A of the water-filled expansion chamber muffler.

Table 1. Material physical properties and corresponding sound speed.

\begin{tabular}{cccccc}
\hline Material & $\mathrm{E}(\mathrm{GPa})$ & $v(1)$ & $\rho\left(\mathrm{kg} / \mathrm{m}^{3}\right)$ & $c_{l}(\mathrm{~m} / \mathrm{s})$ & $c_{s}(\mathrm{~m} / \mathrm{s})$ \\
\hline Water & - & - & 1000 & 1500 & - \\
Aluminum & 68.5 & 0.34 & 2700 & 6248 & 3077 \\
Steel & 210 & 0.30 & 7800 & 6020 & 3218 \\
Perspex & 53.5 & 0.35 & 1200 & 8459 & 4064 \\
Copper & 123 & 0.35 & 8900 & 4710 & 2262 \\
\hline
\end{tabular}

When the acoustic-structural coupling effect is not considered, the cut-off frequency $\left(f_{\text {cut }-o f f}\right)$ of the muffler outlet pipe can be calculated:

$$
f_{\text {cut }- \text { off }}=\frac{3.832}{\pi d} c_{w}=36594 \mathrm{~Hz}
$$

It is recommended by LMS Virtual Lab that the smallest wave-length should be resolved by at least six elements. Since the longitudinal wave speed is greater than the transverse wave speed in a solid, the maximum edge length $\left(L_{\max }\right)$ of the element should comply the following condition:

$$
L_{\max }={ }^{c_{l}} / 6 f_{\max }
$$

In this study, the maximum side length of the elements is limited to $1 \mathrm{~mm}$ in solids, and the maximum analysis frequency can reach $471 \mathrm{kHz}$. Similarly, the maximum elements side length is set to $10 \mathrm{~mm}$, and the maximum analysis frequency can reach to $25 \mathrm{kHz}$.

\subsubsection{Effect of elastic wall thickness}

All elastic walls are made of aluminum, and the acoustic-solid coupling effect is considered. Not only that the inlet and outlet of the muffler are set as fixed constraints, in this study. In order to investigate the influence of wall thickness on the TL of expansion chamber muffler, The four groups based on wall thickness are represented as $1 \mathrm{~mm}(\mathrm{~S} 1), 2 \mathrm{~mm}(\mathrm{~S} 2)$, $5 \mathrm{~mm}(\mathrm{~S} 3), 10 \mathrm{~mm}(\mathrm{~S} 4)$, respectively. It is significant to note that a control group that does not consider the acoustic-structure coupling effect is defined, and it is marked as "SO".

The frequency band of interest is $60-6000 \mathrm{~Hz}$, and the calculation step is $60 \mathrm{~Hz}$. The TL of the model is calculated, as shown in Figure 2. Particularly, the passing frequency $f_{n}^{\text {pass }}$ and maximum noise attenuation $T L_{\text {max }}$ of the expansion chamber muffler can be calculated using plane wave theory, which is the control group "S0", the acoustic-structure coupling effect is should not considered.

$$
f_{n}^{\text {pass }}=\frac{n c_{w}}{2 L}(n=1,2,3 \cdots)
$$




$$
T L_{\max }=10 \lg \left[1+\frac{1}{4}\left(\left(\frac{D}{d}\right)^{2}-\left(\frac{d}{D}\right)^{2}\right)^{2}\right]
$$

In addition, the frequency point $f_{n}^{T L m a x}$ corresponding to the maximum transmission loss $T L_{\max }$ can be obtained:

$$
f_{n}^{\text {TLmax }}=(2 n-1) \frac{c_{w}}{4 L}(n=1,2,3 \cdots)
$$

Therefor, $f_{n}^{\text {pass }}=3000 n \mathrm{~Hz}, T L_{\max }=13.17 \mathrm{~dB}$, and $f_{n}^{T L m a x}=1500(2 n-1) \mathrm{Hz}$. This is in good agreement with the numerical calculation results of "S0" in this study, so the correctness of the acoustic performance of the water-filled muffler under the rigid condition of the model has been verified, as shown in Figure 2. By comparing the numerical re-

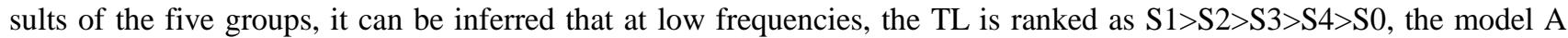
with the smallest elastic wall thickness has the largest TL, on the contrary, S0 that does not consider the acoustic-structure coupling effect, whose TL is the smallest. For the model A, the first order passing frequency shows a distinct difference from that of the control group (S0), which is influenced by the interaction between water and solid. To summarize this phenomenon, the thinner the elastic wall, the lower the first-order natural frequency, and the lower the first order passing frequency. However, the increase in the thickness of the elastic wall reduces the average TL, as shown in Figure 3.

This means that it is indispensable to discuss the acoustic-structure coupling effect when considering the TL of a water-filled muffler.

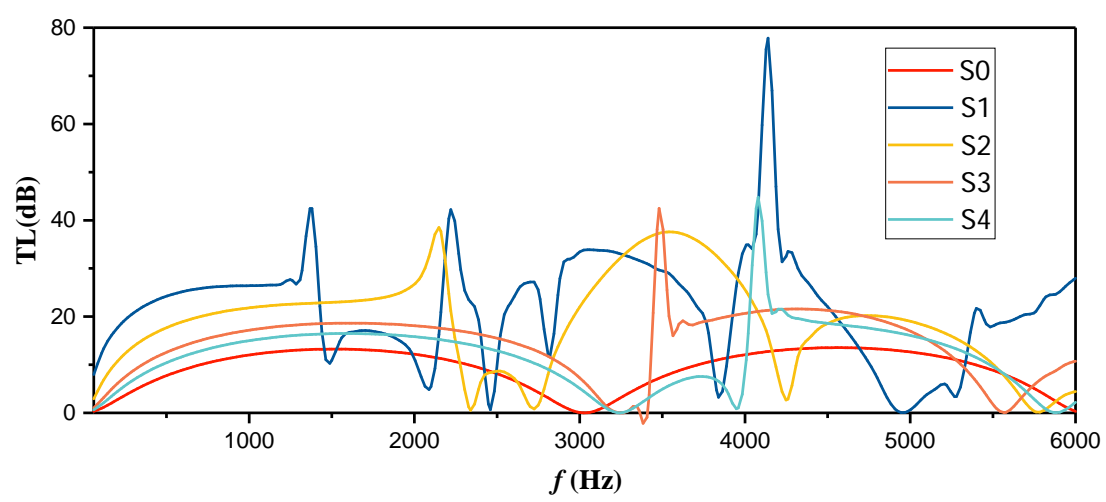

Figure 2. Effects of the wall thickness of circumferential chamber on the TL.

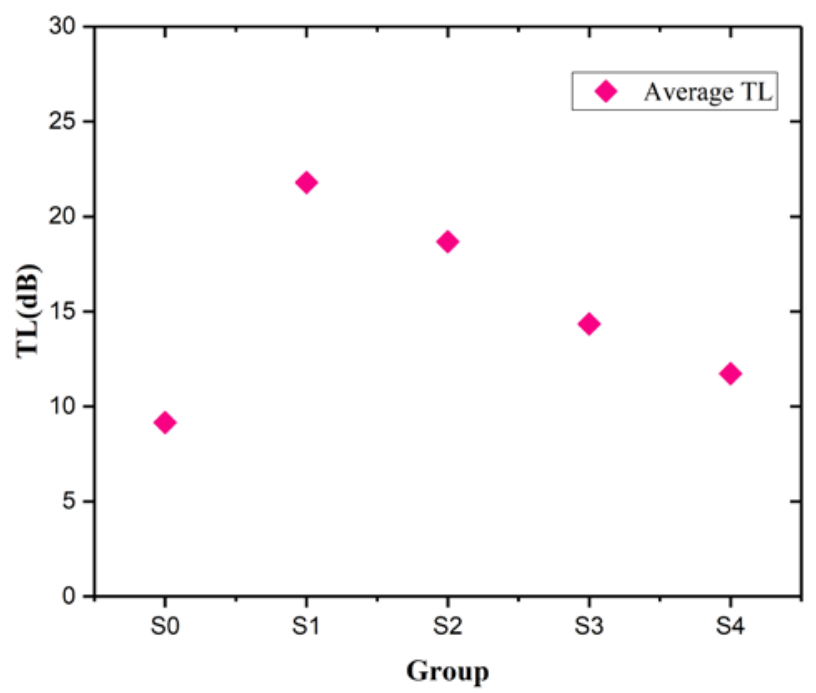

Figure 3. Average TL of model A with different wall thickness. 


\subsubsection{Effect of elastic wall property}

Similarly, Model A was used to study the effects of the elastic wall on the acoustic characteristics of filled water-filled muffler. Assuming that the thickness of the elastic wall is set to $1 \mathrm{~mm}$, and the boundary conditions are the same as in Section 3.1.1, the TL of mufflers with different material properties is investigated. The numerical results are shown in Figure 4.

Figure 4 is a comparison diagram of the TL curves of four different material muffler models. It can be concluded that the TL of the water muffler of the four different materials at low frequencies is ranked as: Perspex > Aluminum> Copper $>$ Steel, the TL of the Perspex model is the largest, while the TL of the Steel model is the smallest. The elastic modulus of the material in this example: Perspex < Aluminum < Copper < Steel, it can be inferred that the smaller the elastic modulus of the elastic chamber wall, the stronger the acoustic-structural coupling effect, for model A. Moreover, the low frequency trend of the first peak position is related to the attenuation of the elastic modulus. It cannot be ignored that the enhancement of the acoustic-structure coupling effect induces the increase of the average TL in the $2000-6000 \mathrm{~Hz}$ frequency band, as shown in Figure 5.

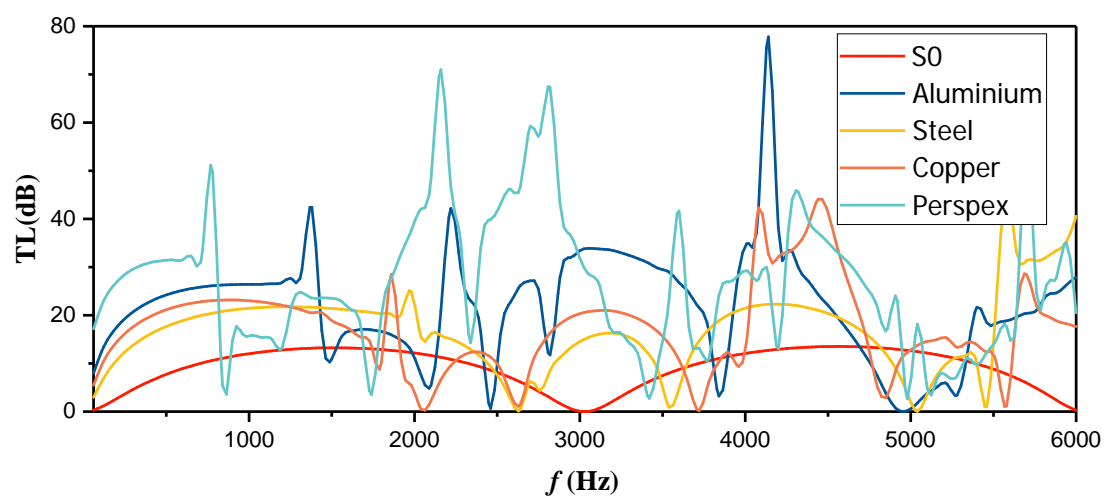

Figure 4. Effects of the wall properties of circumferential chamber on the TL.

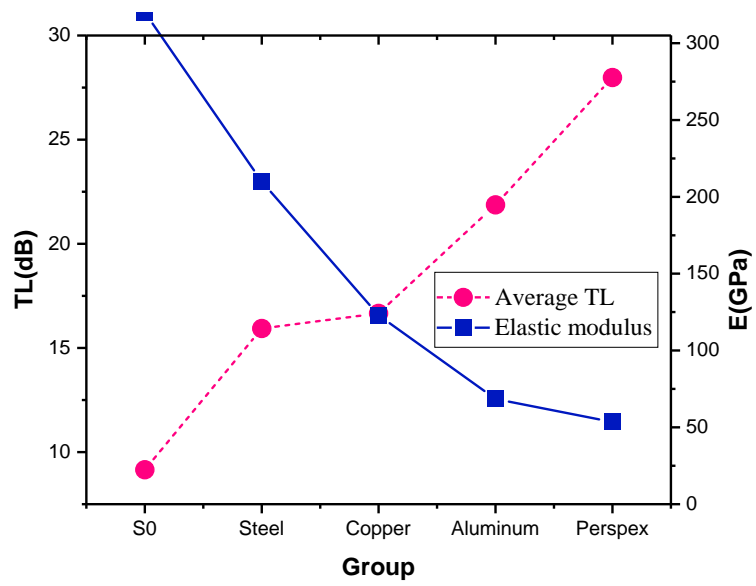

Figure 5. Correlation comparison between elastic modulus and average TL.

\subsection{Water-filled perforated plate muffler}

\subsubsection{Effect of elastic perforated plate thickness}

When the acoustic-structural coupling effect is not considered, the acoustic characteristics of the perforated plate can be replaced by the perforated acoustic impedance, and further some empirical formulas that are in good agreement with the actual engineering are widely used. Since perforations are no longer built in the model, numerical settlement is simplified. However, the coupling between the perforated plate and the fluid is specially considered in this study, so the empirical formula is no longer applicable. Therefore, all perforations are constructed in the model, as shown in Figure 6 (model B). Model B has a total length of $\mathrm{L}=160 \mathrm{~mm}$, a square with a width of $\mathrm{W}=60 \mathrm{~mm}$ at the inlet and outlet ends, and a perforated plate with a wall thickness of $t \mathrm{~mm}$. It should be noted that the inlet and outlet circumferential walls of Model $\mathrm{B}$ are assumed to be rigid walls. 


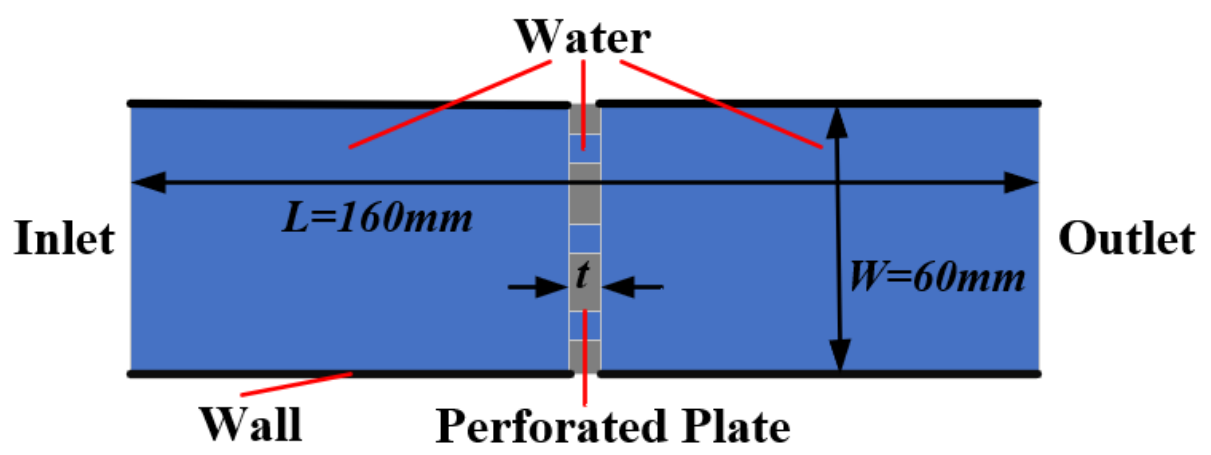

Figure 6. Geometry model B of the water-filled perforated plate muffler.

When the hole diameter of the perforation is set to $r=5 \mathrm{~mm}$, the distance between the centers of two adjacent holes is $\mathrm{l}=20 \mathrm{~mm}$, and the arrangement of the holes is a square arrangement. There are 9 holes in the perforated plate, so the perforation rate is $4.9 \%$. To study the influence of the perforated plate thickness of acoustic performance, the Model B assumed perforation rate and other boundary conditions does not change.

The frequency band of interest is $200-16000 \mathrm{~Hz}$, and the calculation step is set to $200 \mathrm{~Hz}$. Four groups of perforated plate thicknesses of 1(T1), 2(T2), 5(T3), and $10 \mathrm{~mm}(\mathrm{~T} 4)$ are calculated. The results are shown in Figure 7.

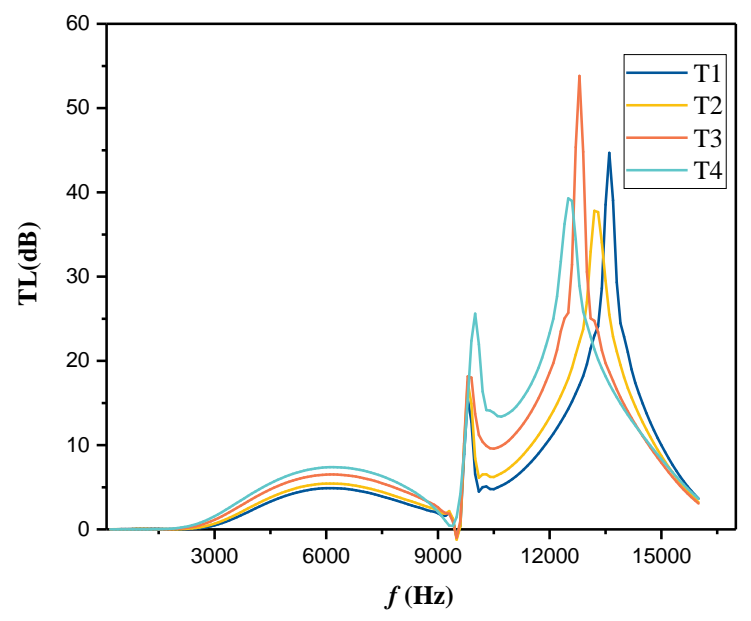

Figure 7. Effects of the wall thickness of perforated plate on the TL of water-filled perforated plate mufflers.

It can be seen from Figure 7 that the TL of water-filled perforated plate mufflers of different thicknesses are closely related to the frequency. Obviously, there is almost no difference in the TL curve below 2000 Hz. However, the thicker the perforated plate, the greater the TL, in the $2000-10000 \mathrm{~Hz}$ frequency band. It is worth noting that comparing several curves with the first resonance peak around $10000 \mathrm{~Hz}$, the thicker the perforated plate, the later and higher the resonance peak appears, that is, the higher the first resonance frequency and the greater the corresponding TL. In contrast, the second resonance peak near $13000 \mathrm{~Hz}$ is observed and the thicker the perforated plate, the earlier the resonance peak appears. According to these four TL curves, it can be seen that the wall thickness of the perforated plate does not seem to have significant influence on the acoustic characteristics of the muffler.

\subsubsection{Effect of Perforation rate}

Assuming that the previous acoustic boundary conditions of the model do not change, the effect of the perforation rate on the acoustic characteristics of the muffler will be investigated. The cross-sections of the four grouped model perforated plates are shown in Figure 8. The diameters of the small holes are all $5 \mathrm{~mm}$, the number of small holes are $4 \mathrm{~mm}$, $9 \mathrm{~mm}, 16 \mathrm{~mm}$ and $25 \mathrm{~mm}$, and the center distances of two adjacent small holes are $30 \mathrm{~mm}, 20 \mathrm{~mm}, 15 \mathrm{~mm}$ and $12 \mathrm{~mm}$ respectively. The perforation rates of the corresponding models are $2.2 \%, 4.9 \%, 8.7 \%$ and $13.6 \%$ respectively. The numerical calculation results are shown in Figure 9. 


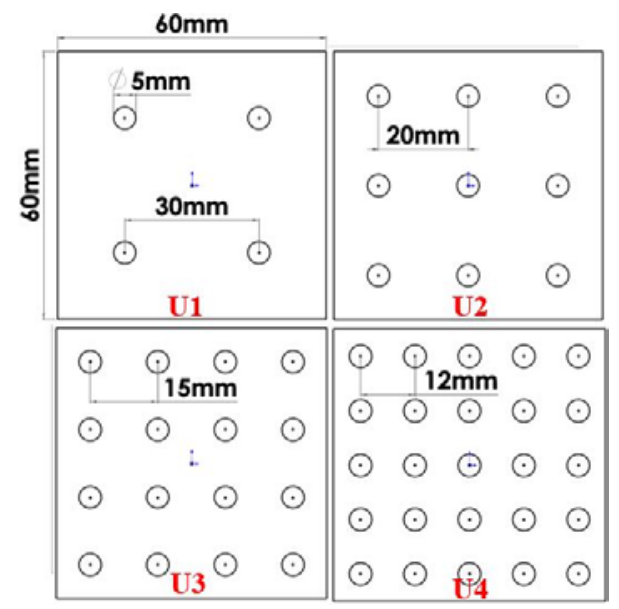

Figure 8. Sketches of penetration distribution based on different penetration rates.

This is an insignificant effect of the change in the perforation rate on the acoustic performance of the perforated plate muffler, within $7000 \mathrm{~Hz}$. An interesting phenomenon is that the perforation rate has a more obvious effect on the high-frequency acoustic characteristics, which is around $12000 \mathrm{~Hz}$. As the perforation rate increases, the TL peak tends to move at higher frequencies and the peak attenuates. However, the reduction of the perforation rate may change the low-frequency acoustic performance, which also means an increase in flow resistance.

In short, the perforation rate is an important factor influencing the acoustic characteristics compared to the thickness of the perforated plate, considering the acoustic-structure coupling.

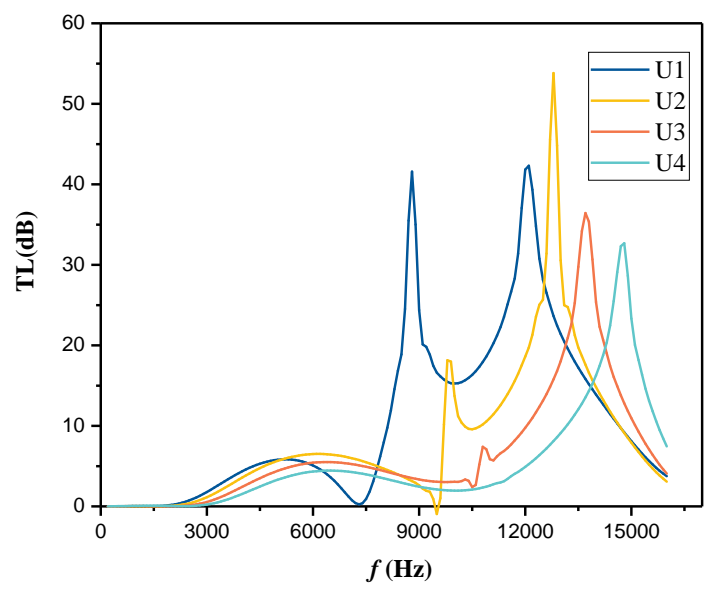

Figure 9. Effects of the Perforation rate on the TL of water-filled perforated plate mufflers.

\section{3 water-filled perforated pipe muffler}

The expansion chamber muffler and the perforated pipe constitute the perforated pipe muffler. This section mainly investigates the acoustic characteristics of the perforated tube muffler, under the condition of acoustic-structure coupling. The calculation Model C is shown in Figure 10. The diameter and length of the expansion chamber of model $\mathrm{C}$ are set as $\mathrm{D}=110 \mathrm{~mm}$ and $\mathrm{L}=200 \mathrm{~mm}$, respectively. The length of the inlet and outlet pipes are both $\mathrm{l}=125 \mathrm{~mm}$, and the acoustic-structure coupling effect of the inlet and outlet pipes is ignored. Perforated pipe inner diameter D1, outer diameter $\mathrm{D} 2=36 \mathrm{~mm}$. When the diameter of the perforated circular hole is assumed to be $6 \mathrm{~mm}$, the perforation rate of the perforated pipe can be determined to be $9 \%$, and 66 perforations are evenly distributed in the perforated pipe.

In order to better investigate the effect of elastic walls at different locations on the acoustic performance, the elastic walls were set as end wall (EW), circumferential wall (CW) and perforated pipe wall (PW). Some default parameters need to be specified in advance. The material of the muffler is steel and the initial thickness of all elastic walls is $\mathrm{t}=2 \mathrm{~mm}$ unless otherwise specified. Other than that, the acoustic boundary conditions are consistent with the previous section. 


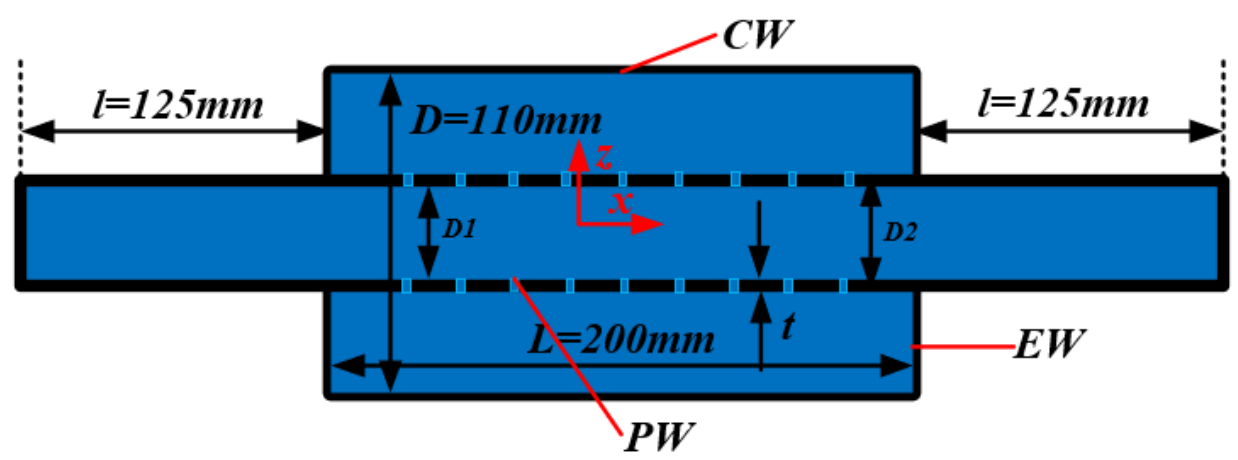

Figure 10. Geometry model B of the water-filled perforated pipe muffler.

According to whether to consider the elasticity of the following three groups of walls: end wall, circumferential wall, and perforated pipe wall, some group numbers are set to express brief descriptions.U1 is adopted to represent the model when only the elasticity of CW is considered. Similarly, U2 and U3 represent the elasticity of PW or EW, respectively. For model C, a group that does not consider the acoustic-structure coupling effect at all is set as U0.

Figure 11 shows the comparison of the TL of the four groups with considering the elasticity of the wall.

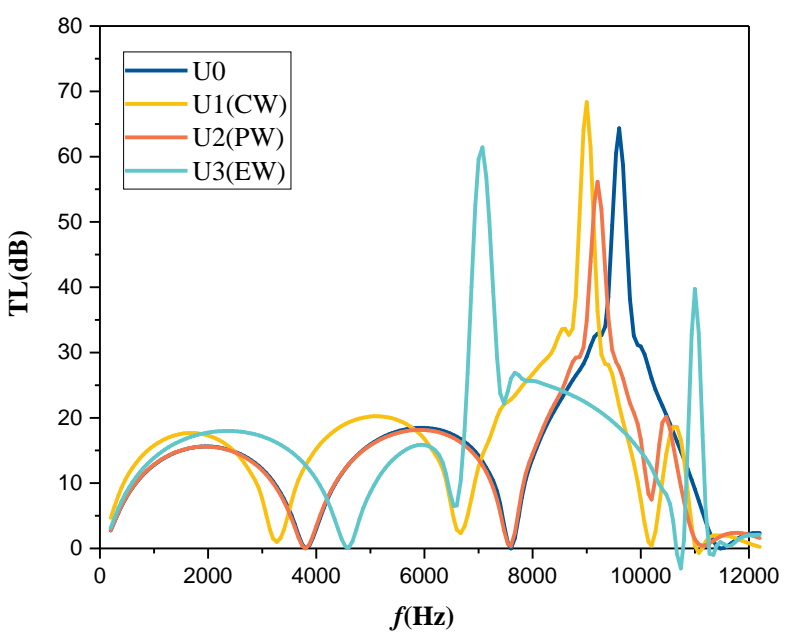

Figure 11. Effects of the wall thickness of perforated plate on the TL of water-filled perforated pipe mufflers.

It can be clearly seen that, the location of the elastic walls affects the pass frequencies and peak frequency in the TL curves, that is to say, the location of elastic walls affects the axial modes and transversal modes of the expansion chamber. Above the first higher order transversal modal frequency, the periodic domes disappear, and the peak appears. The sound propagation field is three-dimensional, and the acoustic attenuation performance is affected by several factor. Hence, only the acoustic characterizes below the peak frequency in the TL curves are analyzed. From Figure 11, it can be seen that, compared with the TL curve of reference group (U0), which does not consider the elasticity, the elasticity of EW (U3) has the largest impact on the pass frequencies and peak frequency, the elasticity of CW (U1) takes the second place, and the elasticity of PW (U2) has the largest impact. Similarly, below the peak frequencies, the TL curve (U0) as almost coincides with the TL curve (U2). On the contrary, it is quite different from the TL curves (U1) and (U3). A conclusion can be inferred that the contribution of EW to acoustic-structure coupling is greater than that of CW, and the contribution of PW is the smallest. To a certain degree, the effects of the elasticity of PW can be ignored. The reason is that compared with the dimension of circumferential wall, the perforated tube wall size is small, and the fluid flow direction is parallel to the perforated tube, which has less impact on the perforated tube wall. However, the fluid directly impacts the end wall, so the sound-structure coupling is obvious. Obviously, when the elasticity of all walls is considered, the calculation result is closest to the real value. In the simulation calculation, the elasticity of perforated tube wall can be ignored, which can greatly simplify the calculation model and improve the calculation efficiency. 


\section{Conclusions}

In this paper, the regular patterns of the TL of three liquid-filled mufflers are investigated, using Virtual Lab, by changing the thickness and material of the elastic wall, the structural parameters of the perforated plate.

1. When the thickness of the circumferential chamber wall becomes thinner or the elasticity is weakened, the peak of the TL curve of thewater-filled expansion chamber muffler moves to the low frequency and the average value increases, due to the enhancement of the acoustic-structure coupling effect.

2. Perforation rate is the primary influencing factor on the TL of perforated plate muffler. The increase of the perforation rate causes the first-order pass frequency to move to high frequency, on the contrary the influence of wall thickness can be ignored.

3. The least impact on the TL of the water-filled perforated pipe mufflers is the elasticity of the PW. When the elasticity of EW is not neglected, the first-order pass frequency of the muffler moves to high frequency, and the peak position has changed greatly.

\section{Acknowledgements}

The authors acknowledge the support of the fundamental research funds for the central universities, HUST: 2018KFYYXJJ013.

\section{References}

[1] Cheng, G. F., Zhang, W. P., Liu, G. M., et al. (2004). The Liquid-born Noise and Its Control in Water Pipelines of Ships. Noise and Vibration Control, 31-33(24).

[2] Wu, Dazhuan, Liu, et al. (2015). Acoustic performance of a water muffler. Noise Control Engineering Journal, (63): 239-248.

[3] Gong, J. F., Xuan, L. K., Peng, C. M. (2019). Analysis of acoustic characteristics of the expansion chamber water muffler considering acoustic-structure interaction. Noise Control Engineering Journal, 4(67): 270-281.

[4] Yuan, S. W., Zhu, H. C., Hou, J. X. (2021). Finite element analysis of perforated tube water muffler with flexible dorsal cavity. Ship Science and Technology, 2(43): 76-79.

[5] Ross, D. F. (1981). A finite element analysis of perforated component acoustic systems. Journal of Sound and Vibration, 1981, 79(1): 133-143.

[6] Fang, Z., Ji, Z. L. (2012). Finite element analysis of transversal modes and acoustic attenuation characteristics of perforated tube silencers. Noise Control Engineering Journal, 3(60): 340-349.

[7] Munjal, M. L., Rao, K. N., Sahasrabudhe, A. D. (1987). Aeroacoustic analysis of perforated muffler components. Journal of Sound and Vibration, 114(2): 173-188.

[8] Xuan, L. K., Jin, G. Y., Gong, J. F., et al. (2014). Time domain finite volume method for three-dimensional structural—acoustic coupling analysis. Applied Acoustics, 76(feb.): 138-149.

[9] Ji, Z. L., Selamet, A. (2004). Boundary element analysis of three-pass perforated duct mufflers. Noise Control Engineering Journal, 48(5): 151-156.

[10] T. W, Wu, et al. (1998). Boundary element analysis of mufflers with an improved method for deriving the four-pole parameters. Journal of Sound \& Vibration.

[11] Liu, C., Ji, Z. L., Fang, Z. (2013). Numerical analysis of acoustic attenuation and flow resistance characteristics of double expansion chamber silencers. Noise Control Engineering Journal, 61(5): 487-499.

[12] Fang, Z., Liu, C. Y. (2017). Combined mesh free method and mode matching approach for transmission loss predictions of expansion chamber silencers. Engineering Analysis with Boundary Elements, 84: 168-177.

[13] Xu Zhou, Zhenlin Ji. (2016). Sound attenuation analysis of water-filled perforated pipe silencers using three-dimensional time-domain computational fluid dynamics approach. Advances in Mechanical Engineering.

[14] Brunner, D., Junge, M., Gaul, L. (2009). A comparison of FE-BE coupling schemes for large-scale problems with fluid—structure interaction. International Journal for Numerical Methods in Engineering, 77(5): 664-688.

[15] Lyon, R. H. (1963). Noise Reduction of Rectangular Enclosures with One Flexible Wall. The Journal of the Acous- 
tical Society of America, 35(11): 1791-1797.

[16] Gladwell, G. (2001). On the reconstruction of a damped vibrating system from two complex spectra. Journal of Sound and Vibration.

[17] Norris, A., Wickham, G. (1993). Elastic Helmholtz resonators. Journal of the Acoustical Society of America, 92(4): 2454.

[18] Zhou, C. (2007). Effect of elastic cavity walls on acoustic characteristics of a water-filled Helmholtz resonator: equivalent lumped parameter model for cylindrical cavity. Acta Acustica., (32): 426-434.

[19] He, T., Sun, G., Sun, Y. D. (2014). Parameter analysis on hydrodynamic noise plate-silencer with fluid cavity. Journal of Ship Mechanics, 4(18): 459.

[20] Gong, J. F., Xuan, L. K., Zhou, J., et al. (2018). Effects of acoustic solid interaction on acoustic characteristics of water expansion chamber muffler. Journal of Harbin Institute of Technology, 189-193(50).

[21] Chen, Liu, Zhenlin, et al. (2014). Computational Fluid Dynamics-Based Numerical Analysis of Acoustic Attenuation and Flow Resistance Characteristics of Perforated Tube Silencers. Journal of vibration and acoustics: Transactions of the ASME. 136(2): 21001-21006. 